\title{
CARACTERIZAÇÃO DA PASSAGEM DE PLANTÃO ENTRE O CENTRO CIRÚRGICO E A UNIDADE DE TERAPIA INTENSIVA
}

\author{
Bárbara Ribeiro Miquelin Bueno¹, Suzana Soares de Moraes², Karina Suzuki³, Fernanda Alves Ferreira Gonçalves ${ }^{4}$, Regiane \\ Aparecida dos Santos Soares Barreto ${ }^{5}$, Cyanéa Ferreira Lima Gebrim ${ }^{6}$
}

${ }^{1}$ Enfermeira. Especialista em UTI. Universidade Federal de Goiás. Goiânia, GO, Brasil.

${ }^{2}$ Enfermeira. Residente em Enfermagem em UTI. Universidade Federal de Goiás. Goiânia, GO, Brasil.

${ }^{3}$ Enfermeira. Doutoranda em Enfermagem. Docente de Enfermagem da Universidade Federal de Goiás. Goiânia, GO, Brasil.

${ }^{4}$ Enfermeira. Doutoranda em Enfermagem. Universidade Federal de Goiás. Goiânia, GO, Brasil.

${ }^{5}$ Enfermeira. Doutora em Enfermagem. Docente de Enfermagem da Universidade Federal de Goiás. Goiânia, GO, Brasil.

${ }^{6}$ Enfermeira. Doutoranda em Ciências da Saúde. Universidade Federal de Goiás. Goiânia, GO, Brasil.

RESUMO: A passagem de plantão deve conter todas as informações indispensáveis para continuidade da assistência. Falhas nesse processo podem prejudicar a segurança do paciente, levando a um tratamento inadequado e com potencial perigo. O objetivo deste estudo foi caracterizar a passagem de plantão entre o Centro Cirúrgico e a Unidade de Terapia Intensiva. Estudo descritivo e transversal, realizado na Unidade de Terapia Intensiva adulto de um hospital escola no município de Goiânia - Goiás, no período de maio a agosto de 2014. Os dados foram coletados por meio de um checklist dividido em quatro categorias contendo informações referentes à identificação, procedimento realizado, condições clínicas e cuidados pós-operatórios. A maioria das informações do checklist não foi repassada para a equipe da Unidade de Terapia Intensiva. Espera-se que os dados apresentados contribuam para criação de estratégias seguras e fortaleçam o comprometimento dos profissionais com a qualidade das informações transmitidas durante a passagem de plantão.

DESCRITORES: Comunicação; Enfermagem; Segurança do paciente; Cuidados intensivos.

\section{CHARACTERIZATION OF HANDOVER FROM THE SURGICAL CENTER TO THE INTENSIVE CARE UNIT}

\begin{abstract}
The handover process must contain all the necessary information for continuing care. Ineffective handover process, with loss of information can be a risk to patient safety. The present study aimed to characterize the handover process from the Surgical Center and the Intensive Care Unit. Descriptive cross-sectional study conducted at an adult Intensive Care Unit of a teaching hospital in the city of de Goiânia - Goiás, from May to August 2014. Data was collected through a checklist divided into four categories containing information related to patient identification, procedure performed, clinical status and postoperative care. A great deal of information of the checklist was not transferred to the Intensive Care Unit staff. We expect that the data provided here contribute to the creation of safe strategies and strengthen the commitment of health professionals with the quality of the information transmitted during the handover process.
\end{abstract}

DESCRIPTORS: Communication; Nursing; Patient Safety; Intensive Care.

\section{CARACTERIZACIÓN DEL CAMBIO DE GUARDIA ENTRE EL CENTRO QUIRÚRGICO Y LA UNIDAD DE TERAPIA INTENSIVA}

RESUMEN: El cambio de guardia debe contener todas las informaciones indispensables para continuidad de la asistencia. Errores en ese proceso pueden perjudicar la seguridad del paciente, Ilevando a un tratamiento inadecuado y con potencial peligro. El objetivo de este estudio fue caracterizar cambio de guardia entre el Centro Quirúrgico y la Unidad de Terapia Intensiva. Estudio descriptivo y transversal, realizado en la Unidad de Terapia Intensiva de adultos de un hospital escuela en municipio de Goiânia Goiás, en el periodo de mayo a agosto de 2014. Los datos fueron obtenidos por medio de un checklist dividido en cuatro categorías que contienen informaciones referentes a identificación, procedimiento realizado, condiciones clínicas y cuidatos posoperatorios. La mayoría de las informaciones del checklist no fue comunicada al equipo de la Unidad de Terapia Intensiva. Es esencial que los datos presentados puedan contribuir para la creación de estrategias seguras y fortalezcan el comprometimiento de los profesionales con la cualidad de las informaciones comunicadas durante el cambio de guardia.

DESCRIPTORES: Comunicación; Enfermería; Seguridad del Paciente; Cuidados Intensivos. 


\section{INTRODUÇÃO}

A passagem de plantão relaciona-se com o processo de passar informações específicas sobre o paciente e pode ocorrer em muitos contextos, desde a sua admissão, entre médicos, entre a enfermagem na troca de turno, transferência entre unidades, pós-operatório, e até a alta para casa ou para outra unidade/estabelecimento ${ }^{(1)}$.

Ressalta-se que a comunicação efetiva na passagem de plantão deve conter todas as informações indispensáveis para continuidade da assistência, e devem ser transmitidas de forma clara, precisa, objetiva, evitando-se más interpretações e garantindo a passagem de plantão eficaz ${ }^{(2)}$.

Lacunas na comunicação podem causar danos graves na continuidade dos cuidados, tratamento inadequado, e potencial perigo para o paciente, e são consideradas uma preocupação internacional $^{(3)}$.

Prejuízos decorrentes da falha na comunicação foram as principais causas de eventos sentinela ${ }^{(4)}$. Eventos sentinela são eventos graves indesejáveis que ocorrem em instituições com acreditação e causam comprometimento ao paciente ou aos seus acompanhantes com ou sem sequelas, mostrando que a qualidade do serviço precisa ser melhorada, e que existem falhas estruturais ou assistenciais que estão causando danos aos usuários. Os eventos adversos referem-se a erros médicos, complicação, incidentes com ou sem danos para o paciente devido a falhas organizacionais, humanas ou técnicas ${ }^{(5)}$.

Dos 25000 a 30000 eventos adversos evitáveis que levaram à incapacidade permanente na Austrália, $11 \%$ foram devido a problemas de comunicação, em contraste com $6 \%$ devido à inabilidade técnica dos profissionais de saúde ${ }^{(6)}$.

Em parte, os problemas da passagem de plantão estão enraizados na maneira como os profissionais de saúde são formados ou não (em habilidades de treinamento da equipe e da comunicação), na falta de bons modelos, e num sistema de saúde que promove a autonomia e o desempenho individual ${ }^{(7)}$.

A comunicação entre a equipe de enfermagem faz com que a assistência seja humanizada, pois qualifica a relação entre profissionais e $\mathrm{o}$ paciente. A enfermagem deve ser estimulada e comprometida com a comunicação, entendendo o cliente como sujeito do cuidado e não passivo a ele ${ }^{(8)}$.

A Unidade de Terapia Intensiva (UTI) é uma unidade complexa, com a finalidade de fornecer aos pacientes graves suporte de vida avançado e adequado, segundo as suas necessidades terapêuticas ${ }^{(9)}$. Pacientes submetidos a procedimentos cirúrgicos, principalmente os de grande porte, são encaminhados para a UTI, visando a recuperação anestésica e sua estabilização hemodinâmica ${ }^{(10)}$.

Transferências intra-hospitalares de pacientes cirúrgicos acontecem frequentemente entre a UTI e o Centro-Cirúrgico (CC) e oferecem potencial risco para o paciente, seja durante o transporte ou mesmo durante a passagem do plantão, na troca e aquisição das informações ${ }^{(11)}$.

De acordo com a literatura, a passagem de plantão pode ser realizada de quatro formas: verbal, escrita, fita gravada ou transferência na cabeceira do leito. No entanto, não existe um método superior ao outro, cabe adequar a realidade da unidade e dos pacientes de maneira a garantir a continuidade de cuidados e a segurança do paciente ${ }^{(12)}$.

A segurança do paciente é um desafio global. Tendo em vista a necessidade da criação de medidas para minimizar o impacto dos danos causados aos usuários dos sistemas de saúde, em 2004 a Organização Mundial de Saúde (OMS) lançou a Aliança Mundial para a Segurança do Paciente, com o objetivo de reforçar o compromisso político em prol da melhoria na assistência $^{(13)}$.

A cada dois anos, a Aliança propõe desafios globais a fim de promover e despertar os estados membros sobre temas e áreas evidenciadas como de risco e expressivas. Em 2009, foi lançado o segundo desafio para segurança do paciente, intitulado "Cirurgias Seguras Salvam Vidas", que foca a atenção na prática de cirurgia segura. Um dos objetivos desse desafio é a comunicação efetiva entre a equipe e a troca de informações para garantia da segurança do paciente ${ }^{(14)}$.

A Joint Commission International (JCI) também propôs como nova meta para alcançar a segurança do paciente, a melhoria e efetividade da comunicação entre os profissionais envolvidos na assistência(15).

A implementação da cultura de segurança nos estabelecimentos de saúde é uma ferramenta que auxilia os gestores no fortalecimento das práticas seguras. Ela pode ser caracterizada 
como um conjunto de regras, rotinas, avaliações e percepções comuns entre os profissionais das unidades de saúde. Além de estabelecer como os profissionais trabalham em equipe $\mathrm{e}$ também como os mesmos se relacionam e se comunicam ${ }^{(16)}$.

Frente a essas questões, os estudos em segurança do paciente e gerenciamento de risco propõem medidas que podem ser aliadas no processo de trabalho a fim de facilitar e sistematizar a comunicação e os cuidados prestados ao cliente. Como uma das medidas, é proposta a criação de protocolos e checklists para aumentar a segurança na comunicação(11).

Protocolos são ferramentas utilizadas para uniformizar procedimentos, aumentar eficácia, mensurar melhorias, diminuir erros, e não deixar que nada seja esquecido, apenas otimizado ${ }^{(16)}$. Os protocolos melhoram a comunicação e permitem maior colaboração da equipe, pois passam a compartilhar fins comuns ${ }^{(9)}$.

Associados aos protocolos encontram-se os checklists que são ferramentas utilizadas para auxiliar na realização de rotinas complexas, aumentando a segurança, diminuindo gastos e otimizando o tempo da equipe ${ }^{(16)}$.

Durante a transferência do paciente do CC para a UTI, há informações que não devem ser perdidas, pois são fundamentais para a continuidade da assistência e segurança do paciente, considerando os procedimentos de alta complexidade a que são submetidos e sujeitos a graves intercorrências ${ }^{(17)}$.

A qualidade da passagem de plantão fortalece a prática segura das ações e fornece embasamentos para os cuidados de enfermagem. A criação de um instrumento poderá auxiliar os enfermeiros nessa transição de informações para que estas não sejam perdidas, diminuindo a possibilidade de falhas na comunicação.

Dessa maneira, o objetivo deste trabalho foi caracterizar a passagem de plantão entre o CC e a UTI.

\section{MÉTODOS}

Estudo descritivo e transversal. Realizado na UTI adulto de um hospital escola no município de Goiânia - Goiás. Foram inclusos no estudo todas as informações recebidas durante a passagem de plantão do CC para a UTI, de segunda a sexta das 7 às 19 horas, no período de maio a agosto de 2014 .
A coleta de dados foi realizada por meio de um checklist pelas pesquisadoras e também por quatro coletadores treinados previamente. Este checklist foi preenchido durante a passagem de plantão, a qual foi realizada via telefone, em que o enfermeiro ou técnico de enfermagem do CC ligava e passava todas as informações referentes ao transoperatório do paciente para a equipe de enfermagem da UTI. O CC fica localizado no mesmo andar e a cerca de 100 metros da UTI do estudo e o tempo gasto para o transporte é em média 10 minutos. Foram excluídos os cheklists de pacientes provenientes do centro obstétrico, da unidade de hemodinâmica, do pronto socorro e das unidades clínicas.

O checklist foi dividido em quatro categorias. Categoria 1: identificação do paciente (nome, idade, diagnóstico primário e procedimento cirúrgico); categoria 2: procedimento cirúrgico realizado (intercorrências durante procedimento cirúrgico); categoria 3: condições clínicas na Sala de Recuperação Pós-Anestésica (SRPA) (uso de oxigênio por cateter, máscara facial, tubo orotraqueal ou traqueostomia), Pressão Arterial Invasiva (PAI), e a presença de drenos; e categoria 4: cuidados pós-operatório (restrição de decúbito (decúbito lateral direito, esquerdo ou dorsal), restrição para aferir pressão arterial não invasiva em membros (membro superior direito ou esquerdo, membro inferior direito ou esquerdo), cuidados com curativos (manutenção do curativo ocluído por 72 horas, utilização de produtos especiais), manutenção de membros aquecidos (membros superiores ou inferiores).

Este estudo faz parte do projeto intitulado: "Promovendo a segurança do paciente no perioperatório" e foi aprovado pelo Comitê de Ética em Pesquisa do Hospital das Clínicas da Universidade Federal de Goiás (CEP/HC/UFG), sob o protocolo n. 018/2011.

As informações obtidas foram organizadas em banco de dados eletrônico, no Microsoft Excel 2010, Windows 2007 e submetidas a uma análise descritiva, mediada por tabelas de frequências absoluta e relativa.

\section{RESULTADOS}

Foram admitidos 90 pacientes dos quais 71 foram possíveis de serem registrados no checklist, contudo 19 foram recebidos na UTI sem a passagem de plantão, sendo assim, excluídos do estudo.

Na categoria 1, identificação do paciente, houve 
maior registro quanto ao nome do paciente, entretanto, a idade e o diagnóstico primário do paciente quase não foram informados. Da categoria 2, foram 06 cheklists que registraram se houve ou não intercorrência durante o ato cirúrgico, sem repassar qual a intercorrência. $\mathrm{Na}$ categoria 3, condições clínicas, a informação mais repassada foi quanto ao uso de oxigênio e uso de PAI. Informação como uso de drogas vasoativas também não foi repassada para a equipe da UTI e houve 01 registro de aviso sobre a presença de dreno. Na categoria 4, não houve nenhum registro referente aos cuidados no pós-operatório.

Dos 71 checklists analisados, 50,7\% informaram o nome do paciente, $42,3 \%$ avisaram sobre a presença ou não de $\mathrm{PAI}, 33,8 \%$ comunicaram sobre o tipo de oxigenoterapia, $29,6 \%$ falaram qual o procedimento realizado no CC, apenas $4,2 \%$ informaram o diagnóstico primário do paciente, $2,8 \%$ sabiam informar a idade e somente $1,4 \%$ informou se havia ou não dreno, porém não sabia informar qual o tipo de dreno (Tabela 1).

Apesar de não fazer parte das informações do checklist, $32,4 \%$ das fichas continham a informações que o paciente iria "extubado" para a UTI, informação que não auxilia a equipe, pois existem várias formas não invasivas de fornecimento de oxigênio. E em 31\% constava em quanto tempo o paciente seria transferido para a UTI.

Vale salientar que a passagem de plantão presencial não é realizada entre os enfermeiros das unidades contidas no estudo. Em todos os casos a passagem de plantão é realizada por telefone e o paciente é transportado pelo condutor de macas e o anestesista do CC para a UTI, que passa o caso para o médico intensivista, e nessa ocasião o enfermeiro coleta as informações adicionais e posteriormente analisa o prontuário.

\section{DISCUSSÃO}

A comunicação na área de saúde é muito complexa e dinâmica, pois contém inúmeras informações utilizadas pela equipe multiprofissional envolvida na assistência. Falhas no processo de comunicação entre as equipes dos serviços de saúde favorecem a ocorrência de $\operatorname{erros}^{(18)}$.

A passagem de plantão é uma atividade que exige dos profissionais comprometimento com a continuidade segura da assistência, e salienta-se que a qualidade dessas informações colabora com a sistematização da assistência de enfermagem.
Tabela 1 - Distribuição dos itens e categorias do checklist, segundo a presença ou não na passagem de plantão. Goiânia, GO, Brasil, 2014

\begin{tabular}{lcccc} 
& \multicolumn{2}{c}{ Sim } & \multicolumn{2}{c}{ Não } \\
\hline Itens do checklist & (n) & $\%$ & (n) & $\%$ \\
\hline Categoria 1 & & & & \\
\hline Nome do paciente & 36 & 50,7 & 35 & 49,3 \\
\hline Idade & 2 & 2,8 & 69 & 97,2 \\
\hline Diagnóstico primário & 3 & 4,2 & 68 & 95,8 \\
\hline Categoria 2 & & & & \\
\hline Procedimento cirúrgico & 21 & 29,6 & 50 & 70,4 \\
\hline Intercorrências & 6 & 8,4 & 65 & 91,6 \\
\hline Categoria 3 & & & & \\
\hline Uso de oxigênio & 24 & 33,8 & 47 & 66,2 \\
\hline Uso de drogas vasoativas & & & 71 & 100 \\
\hline Uso de PA* & 30 & 42,3 & 41 & 57,7 \\
\hline Dreno torácico & 1 & 1,4 & 70 & 98,6 \\
\hline Categoria 4 & & & \\
\hline Restrição de decúbito & & 71 & 100 \\
\hline Restrição para aferir PA** & & 71 & 100 \\
\hline Cuidados com curativo & & 71 & 100 \\
\hline Manutenção de membros aquecidos & 71 & 100
\end{tabular}

*Pressão arterial invasiva **Pressão arterial

Muitas informações são negligenciadas pela equipe durante a passagem de plantão, fato que potencializa a ocorrência de eventos adversos, comprometendo a segurança do paciente.

Falhas na comunicação são responsáveis por $32 \%$ dos erros referentes à administração de medicamentos, admissões emergentes, e passagem de nomes de pacientes errados para o CC nas UTIs ${ }^{(19)}$. Diante disso destaca-se que uma boa passagem de plantão depende da habilidade do profissional que transmite, do tempo disponível e do envolvimento da equipe com a qualidade das informações ${ }^{(20)}$. As informações contidas nesta atividade devem ser realizadas em tempo satisfatório de maneira que não se gaste muito tempo, para não prejudicar o tempo dispendido para o cuidado, mas que agrupe todas as informações necessárias para a continuidade da assistência.

O código de Ética dos profissionais de enfermagem, Resolução COFEN no 311 de 2007, descreve em seu artigo 41 da Seção II que é dever e responsabilidade dos profissionais de enfermagem: "Prestar informações, escritas e verbais, completas e fidedignas necessárias para assegurar a continuidade da assistência ${ }^{(21: 15)}$. 
Com base nos dados coletados evidenciou-se que as transmissões de informações não estão sendo repassadas de forma completa, o que fere o código de ética e compromete a qualidade e continuidade da assistência.

Frente a esse contexto, destaca-se a cultura de segurança com seu conjunto de valores, atitudes, competências e comportamentos comprometidos com a gestão da saúde e da segurança, a fim de substituir a culpa e a punição pelo aprendizado ${ }^{(11)}$.

Entre os principais problemas envolvidos na qualidade da passagem de plantão estão a falta de tempo para a atividade, registros insuficientes e ausência de clareza das informações ${ }^{(20)}$. Estudo realizado na Austrália mostrou que ocorreram 459 incidentes devidos a falhas no processo de comunicação, entre as principais falhas estão: transferência de pacientes sem a passagem de plantão adequada 28,8\% (132), omissões de informações das condições críticas dos pacientes $19,2 \%$ (88), omissões sobre o plano de cuidados $14,2 \%(65)^{(22)}$.

Diante dessa problemática destaca-se que CC e a UTI são setores de alta complexidade que necessitam de maior clareza e detalhamento de suas atividades. No presente estudo, foi evidenciado que a maioria das informações foi passada de forma resumida e incompleta, o que potencializa a ocorrência de erros durante o seguimento dos cuidados de enfermagem.

Falhas na identificação resultam em diversos eventos adversos relacionados à administração de medicamentos, transfusão de sangue e hemoderivados, procedimentos cirúrgicos errados, exames diagnósticos, e entrega de bebês a famílias erradas ${ }^{(23)}$. Houve baixo registro de informações referentes à identificação dos pacientes nos checklists.

A JCl propõe que a identificação dos pacientes nas unidades de saúde seja padronizada, contendo pelo menos dois dados do paciente como, por exemplo, nome e data de nascimento, nunca utilizar como um dos dois dados o número do leito ${ }^{(21)}$.

Durante o procedimento cirúrgico, o paciente está sujeito a falhas. A Segunda Aliança para Segurança do Paciente propõe que seja feita a identificação do paciente, evitem-se danos durante indução anestésica, estejam preparados para perdas sanguíneas e de via aérea, estejam atentos às reações alérgicas, previnam infecções do sítio cirúrgico, impeçam a retenção de compressas cirúrgicas e instrumentais, melhorem a comunicação entre as equipes com a finalidade de se evitar intercorrências no procedimento cirúrgico ${ }^{(14)}$. Há uma grande preocupação com a comunicação sobre essas intercorrências, pois elas não foram repassadas para a UTI, fato evidenciado em apenas seis registros.

Destaca-se que pacientes no pós-operatório podem vir a apresentar vários distúrbios inerentes ao ato cirúrgico, como: distúrbios cardiovasculares, pulmonares e renais, estas disfunções devem ser reconhecidas e tratadas de imediato a fim de evitar complicações no pósoperatório. Assim, a avaliação de enfermagem no pós-operatório deve ter enfoque na segurança do paciente, visando a prevenção de danos desnecessários ${ }^{(24)}$.

Pacientes no pós-operatório devem ser incentivados o mais precocemente possível a se movimentar, porém em alguns casos essa movimentação é restringida devido ao tipo ou extensão da cirurgia ${ }^{(25)}$. Com base nos resultados apresentados, nota-se que muitas informações importantes sobre as condições clínicas e referentes à continuidade da assistência não foram comunicadas aos profissionais de enfermagem da UTI.

Considerando o paciente no transoperatório, a falta de informações pode gerar situações de estresse desnecessárias para a equipe da UTI e do CC e consequentemente demora em planejamento do cuidado.

\section{CONCLUSÃO}

Ressalta-se que a criação de protocolos e instrumentos de passagem de plantão podem auxiliar os enfermeiros a otimizar o tempo e garantir que informações importantes não sejam perdidas durante esse processo. É importante destacar que esses instrumentos devem ser adequados conforme a rotina e a especialidade do setor.

A passagem de plantão do CC para a UTI precisa ser melhorada, pois informações referentes à identificação dos pacientes, às intercorrências ocorridas no transoperatório, alergias prévias, estado clínico, os cuidados pós-operatórios recomendados, não estão sendo repassadas para os profissionais de enfermagem da UTI.

Os dados apresentados permitiram identificar pontos fracos/ falhas no processo de comunicação, os quais permitirão a criação de estratégias seguras à prática desta atividade e 
para o maior comprometimento dos profissionais com a qualidade das informações transmitidas durante a passagem de plantão.

O presente estudo apresentou como limitação o fato da passagem de plantão ser realizada via telefone. Recomenda-se para futuros estudos que a passagem de plantão pela equipe de enfermagem seja realizada presencialmente.

\section{REFERÊNCIAS}

1. World Health Organization (WHO). Communication during patient hand-overs. Patient safety solutions. Joint Commission International. [Internet] 2007;1 solution 3. [acesso em 13 de nov 2013]. Disponível: http://www. who.int/patientsafety/solutions/patientsafety/PSSolution3.pdf

2. World Health Organization (WHO). WHO guidelines for safe surgery. Geneva. 2009. [acesso em 13 de nov 2013]. Disponível: http://whqlibdoc.who.int/ publications/2009/9789241598552_eng.pdf?ua=1

3. Agência Nacional de Vigilância Sanitária (ANVISA). Boletim Informativo: Segurança do paciente e qualidade em serviços de saúde. [Internet] 2011; 1(1)12p. [acesso em 13 de jun 2014] Disponível: http://portal.anvisa.gov.br/wps/wcm/connect/ f72c20804863a1d88cc88d2bd5b3ccf0/BOLETIM+I. PDF?MOD=AJPERES

4. Joint Commission International (JCl). Sentinel Event Data. Root causes by event type. [Internet] 2004-2014. [acesso em 02 de nov 2014] Disponível: http://www. jointcommission.org/assets/1/18/Root_Causes_by Event_Type_2004-2014.pdf

5. Organização Nacional de Acreditação (ONA). Glossário e termos técnicos. [Internet] 2006. [acesso em 28 de jun 2015]. Disponível: http://www.anvisa.gov. $\mathrm{br} / \mathrm{servicosaude/acreditacao/manual/glossario.pdf}$

6. Zinn C. 14000 preventable deaths in Australian hospitals. BMJ [Internet] 1995;310(6993). [acesso em 02 de nov 2014]. Disponível: http://dx.doi.org/10.1136/ bmj.310.6993.1487

7. Sadala MLA, Stefanelli MC. Avaliação do ensino de relacionamento enfermeira-paciente. Rev Latino-Am Enfermagem. [Internet] 1996; (4). [acesso em 13 de jun 2014]. Disponível: http://dx.doi.org/10.1590/S010411691996000700014

8. Broca PV, Ferreira MA. Equipe de enfermagem e comunicação: contribuições de enfermagem. Rev Bras Enferm. [Internet] 2012; 65(1) [acesso em 22 de nov 2013]. Disponível: http://www.scielo.br/pdf/reben/ v65n1/14.pdf

9. Fernandes HS, Pulzi Júnior, Costa Filho R. Qualidade em terapia intensiva. Rev Bras Cli Med. [Internet] 2010;8. [acesso em 13 de nov 2013]. Disponível: http:// files.bvs.br/upload/S/16791010/2010/v8n1/a009.pdf

10. Balsanelli AP, Zanei SSSV, Whitaker IY. Carga de trabalho de enfermagem e sua relação com a gravidade dos pacientes cirúrgicos em UTI. Acta Paul. Enferm. [Internet] 2006; 19(1). [acesso em 13 de nov 2013]. Disponível: http://dx.doi.org/10.1590/S010321002006000100003

11. Wachter RM. Erros na transferência de pacientes e na troca de informações. Compreendendo a segurança do paciente. 2. ed. Porto Alegre: Artmed, p.123-45, 2013.

12. Miller C. Ensuring continuing care: styles and efficiency of the handover process. Australian Journal of Advanced Nursing. 1998; 16(1):23-27.

13. World Health Organization (WHO) .World Alliance for patient safety. Forward programme. [Internet] 2004. [acesso em 13 de nov 2013]. Disponível: http:// www.who.int/patientsafety/en/brochure_final.pdf

14. Organização Mundial da Saúde (OMS). Segundo desafio global para a segurança do paciente: Manual - cirurgias seguras salvam vidas. Rio de Janeiro, 2009. 29p. [acesso em 10 de out2014]. Disponível: http:// bvsms.saude.gov.br/bvs/publicacoes/seguranca paciente_cirurgia_salva_manual.pdf

15. World Health Organization (WHO). WHO/JC. Joint Commission International: Patient identification. WHO Collaborating for Patient Safety Solutions. Patient Safety Solution. [Internet] 2007;(1) solution 2. [acesso em 13 de nov 2013]. Disponível: http://www.who.int/ patientsafety/solutions/patientsafety/PS-Solution2.pdf

16. Réa-Neto A, Castro JEC, Knibel MF, Oliveira MC. GUTIS - Guia da UTI segura. Associação de Medicina Intensiva Brasileira - AMIB. $1^{\circ}$ ed, São Paulo, 2010. [acesso em 13 de nov 2013]. Disponível: http://static. hmv.org.br/wp-content/uploads/2014/07/Orgulho_ GUTIS.pdf

17. Agência Nacional de Vigilância Sanitária (ANVISA). Assistência segura: uma reflexão teórica aplicada à prática. Série: Segurança do paciente e qualidade em serviços de saúde. [Internet] 2013;172p. [acesso em 13 de nov 2013]. Disponível: http://www20.anvisa.gov. br/segurancadopaciente/images/documentos/livros/ Livro1-Assistencia_Segura.pdf

18. Almeida ACG, Neves ALD, Souza CLB, Garcia JH, Lopes JL, Barros ALBL. Transporte intra-hospitalar de pacientes adultos em estado crítico: complicações relacionadas à equipe, equipamentos e fatores fisiológicos. Acta Paul Enferm. [Internet] 2012; 25(3). [acesso em 13 de jun 2014]. Disponível: http://dx.doi. org/10.1590/S0103-21002012000300024 
19. Pronovost PJ, Thompson DA, Holzmueller CG, Lubomski LH, Dorman T, Dickman F, et al. Toward learning from patient safety reporting systems. Journal of Critical Care. [Internet] 2006; (21). [acesso em 13 de nov 2013]. Disponível: http://www.ncbi.nlm.nih.gov/ pubmed/17175416

20. Silva EE, Campos FL. Passagem de plantão na enfermagem: revisão da literatura. Cogitare enferm. [Internet] 2007; 4(12). [acesso em 13 de nov 2013]. Disponível: http://dx.doi.org/10.5380/ce.v12i4.10077

21. Conselho Federal de Enfermagem. Resolução n. 311 de 08 de fevereiro de 2007. Código de Ética dos Profissionais de Enfermagem. Rio de Janeiro: COFEN; 2007. [acesso em 17 de out 2014]. Disponível: http://se.corens.portalcofen.gov.br/codigo-de-eticaresolucao-cofen-3112007

22. Thomas MJW, Schultz TJ, Hannaford N, Runciman WB. Failures in transition: learning from incidents relating to clinical handover in acute care. J Healthc Qual.[Internet] 2013;35(3). [acesso em 02 de nov 2014]. Disponível: http://dx.doi.org/10.1111/j.19451474.2011.00189.x

23. Joint Commission Internacional (JCI). Patient safety solutions preamble preámbulo a lãs soluciones para la seguridad del pacient mayo 2007. [acesso em 10 de out 2014]. Disponível: http://www.who.int/patientsafety/ solutions/patientsafety/PatientSolutionsSPANISH.pdf

24. Popov DCS, Peniche ACG. As intervenções do enfermeiro e as complicações em sala de recuperação pós-anestésica. Rev. Esc. Enferm. USP. [Internet] 2009; 43(4). [acesso em 22 de nov 2013]. Disponível: http:// dx.doi.org/10.1590/S0080-62342009000400030

25. Stracieri LDS. Cuidados e complicações pósoperatórias. Medicina (Ribeirão Preto). [Internet] 2008; 41(4). [acesso em 02 de nov 2014]. Disponível: ht tp://revista.fmrp.usp.br/2008/VOL41N4/ SIMP_4Cuidados_e_complicacoes-posoperatorias. pdf 